\begin{tabular}{ll}
\hline \hline MINING AND METALLURGY INSTITUTE BOR & ISSN: 2334-8836 (Štampano izdanje) \\
UDK: 622 & ISSN: 2406-1395 (Online) \\
\hline \hline
\end{tabular}

\author{
Nenad Vušović, Milica Vlahović ${ }^{* *}$, Milenko Ljubojev ${ }^{* * *}$, \\ Miljan Vlahovic ${ }^{* * * *}$, Daniel Kržanovic ****
}

\title{
SOFTWARE SOLUTION FOR THE MINE SUBSIDENCE PREDICTION BASED ON THE STOCHASTIC METHOD INTEGRATED WITH THE GIS
}

\begin{abstract}
Mining induced the ground subsidence and consequently, damages on objects above excavation works is a serious problem all over the world. In this paper, a new approach for subsidence and deformation prediction based on the stochastic Pataric-Stojanovic method is presented. Since the calculation using the equations of this method is very complex, the original computer program package with the title MITSOUKO has been purposely designed. The MITSOUKO program enables calculating at any point of the land surface and representing the results owing to the possibility of their integration and further processing in the Geographical Information System (GIS). The components of the GIS are used to realize the spatial analysis.

Keywords: coal mining; surface subsidence; stochastic prediction method; GIS, spatial analysis
\end{abstract}

\section{INTRODUCTION}

Mine surveying in coal mines all over the world was initiated with the underground coal mining- induced land subsidence and building damage in the Ruhr Basin in the middle of the $19^{\text {th }}$ century. Based on the obtained results, different methods for predicting subsidence and deformation parameters, such as the empirical methods, the profile function methods, the influence function methods, and the void diffusion method were developed [4].
Also, numerous theoretical studies were performed using the stochastic method, the elastic methods, and the visco-elastic method as well as the finite-element methods, the boundary element method, and the distinct element method for the ground subsidence and deformations prediction. Most of these methods cover the horizontal and slightly sloped coal seams. The methods for seams with greater slopes are less reliable but with a high professional improvisation. Further

\footnotetext{
* University of Belgrade, Technical Faculty in Bor, Vojske Jugoslavije 12, Bor, Serbia,

Corresponding author's e-mail: nvusovic@tfbor.bg.ac.rs

** University of Belgrade, Institute of Chemistry, Technology and Metallurgy, Karnegijeva 4, Belgrade, Serbia

**** Mining and Metallurgy Institute Bor, Zeleni bulevar 35, Bor, Serbia

**** Public Company Nuclear Facilities of Serbia, Mike Petrovića Alasa 12-14, 11351 Vinča, Belgrade, Serbia

***** This work was financially supported by the Ministry of Education, Science and Technological Development of the Republic of Serbia (Grant Nos. 451-03-68/2020-14/200131, 451-0368/2020-14/200026 and MMI)
} 
more, the numerical methods were used for subsidence modeling and calculating the rock strata movement $[3,4]$. The shape and position of subsidence trough in relation to the excavated area depend on the geological and technological conditions of underground exploitation; for the horizontal coal seams, it is symmetrical, while for inclined it becomes asymmetrical. The available methods reliably prognose both vertical and horizontal subsidence components and offer deriving other displacement factors of movement, such as the tilt, curvature, and horizontal strain.

Prediction of the final subsidence is simpler compared with the displacements induced by sequential mining processes. Also, many factors affect and conduct moving of the rock mass thus making the behavior of overlying strata complicated. Therefore, different idealized media were used for the subsidence prediction in the middle of the 20th century. The stochastic theory of ground movements proved to be a universal method for subsidence prediction [1].

The first application of the stochastic model was in the soil mechanics for sand, gravel and severely cracked ground. Pokrovsky (1929) concluded that the change in pressure due to the concentrated force at the horizontal plane as a function of depth can be represented by the standard Gaussian distribution curve, also known as a normal distribution curve. According to J. Litwiniszyn, with a presumption that the rock mass is a stochastic medium, the stochastic method interprets the displacement of rock massif related to mine subsidence [2].

Geoinformation technologies offer the management of spatial data with their integration in the Geographic information system (GIS) [6,12]. The computer-based analytical methods that realistically simulate subsidence processes are needed for the reliable design of mining layout to minimize the underground excavation influence on the land surface. Integrative modeling, interac tive spatial analysis and understanding different processes are supported by the GIS. Based on the simulation of spatially distributed and time-dependent subsidence processes, innovative thematic maps containing the land-surface properties can be designed [7-11].

\section{STOCHASTIC METHOD BY PATARIC AND STOJANOVIC}

The stochastic Pataric-Stojanovic method applies the mathematical statistics and assumes the multi-layered massif, split by a series of cracks into a large number of elements with movements having stochastic character.

\subsection{Movements in a massif with horizontal seams}

The environment is presented by the symmetrically arranged elements with similar dimensions. Such an area is not real, but the assumption is statistically correct because the pressure change curve in a homogeneous medium is symmetrical, thus elements in the profile must be symmetrical [1]. Owing to this symmetry, the force got by one element is transmitted and equally divided into two parts on which it relies (Figure 1). For simplicity, a unit force is observed, so if the surface layer is marked as zero, the element in this layer receives the entire force, the two elements in the first layer, on which the element from the zero layer relies, receive half of this force. In the second layer, the edge elements receive a quarter, and the middle two quarters of the force, etc. Following the process further, it is noticed that the distribution of forces obeys the binomial law. When $i, j \rightarrow 0$, the binomial distribution asymptotically tends to normal, then the pressure changes according to the law of normal distribution, and can be represented by a Gaussian curve [2]. 


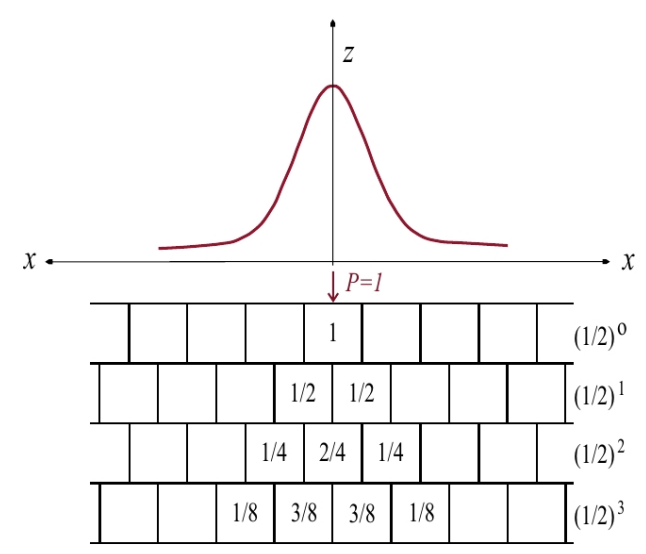

Figure 1 Pressure distribution according to the stochastic model [2]

The mathematical model from Figure 1 can be used to study the plane subsidence of a layered massif [3]. The subsidence will be plane if a long area of constant width ( $2 a)$ and height $(d)$ has been excavated. The assumption is that the rock massif consists of the horizontal layers divided by a series of cracks into equal, symmetrically arranged elements (Figure 2).

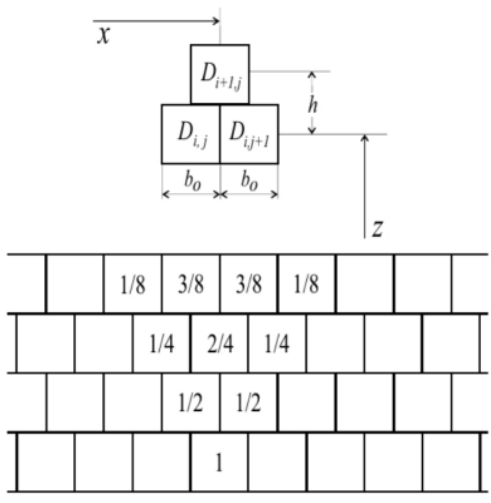

Figure 2 Simmetrically arranged elements in horizontal seams divided by cracks [3]

When the element from the lowest layer is removed, the elements in the higher layers begin to move. Their movement is assumed to be stochastic, so instead of studying the displacement, the probability of the element passing into the lower layer is determined. A place that remains vacant in the lowest layer can be occupied by one of the elements that lie directly above it. Owing to symmetry, the probabilities of these events are the same, so their value equals $1 / 2$. With further following the probabilities for moving elements from higher layers, the same regularity is valid [2]. Since the subsidence is plane, two coordinates are sufficient: an abscissa $x$ (parallel to the layers) and an elevation $z$ (vertically with the upward direction) to determine the position of the elements in the massif. 
For the boundary transition from a discrete division to a continuous massif, the starting point is the position of the elements that are defined by the coordinates, ie:

$$
\begin{aligned}
& F(x, z+h)= \\
& =\frac{1}{2}\left[F\left(x-b_{0}, z\right)+F\left(x+b_{0}, z\right)\right]
\end{aligned}
$$

where $F(x, z)$ is the function of subsidence probability [3].

The assumption is that the excavated area $\Omega_{2}$ has a rectangle shape with width $2 a$ and thickness $d$, so if a coordinate origin is placed in the intersection of rectangle diagonals, it will be:

$$
U(x)=\iint_{\Omega_{2}} F(x, \bar{x}, H, \bar{z}) d \bar{x} d \bar{z}
$$

Since the thickness $d$ is negligible with respect to the depth $H$ of the seam, instead of the integral, its mean value can be taken without a significant error:

$$
\int_{-d / 2}^{d / 2} F(x, \bar{x}, H, \bar{z}) d \bar{z}=d \cdot F(x, \bar{x}, H, 0)
$$

Using the function from the Eq. (1) the subsidence is:

$$
\Phi(t)=\frac{2}{\sqrt{2 \pi}} \int_{0}^{t} e^{-\frac{1}{2} s^{2}} d s
$$

the subsidence is definitely calculated by the formula [3]:

$$
U(x)=U_{0} X_{0}(x)
$$

where:

$$
X_{0}(x)=\frac{1}{2}\left[\Phi\left(\frac{a+x}{n}\right)+\Phi\left(\frac{a-x}{n}\right)\right]
$$

\subsection{Movements in a massif with inclined seams}

In the case of inclined seams, the starting assumption that the massif is divided by a series of horizontal and vertical cracks into elements of the same dimensions. Equation (4) can only be applied to profiles along the strike. However, the subsidence curve is not symmetrical by the seam dip, so there is anisotropy unlike the process in a massif with horizontal seams. The same mathematical model can be used assuming that the elements, with still identical dimensions due to homogeneity, are no longer symmetrically arranged, but the center of each element is shifted by the dip for a certain length $e$, whereby $0<e<b_{1}$ (Figure 3 ). The coordinate origin is placed at an arbitrary point $O$ of the seam that is being excavated, the $z$ axis is directed vertically upwards, the $x$ axis is directed horizontally along the seam strike, and the $y$-axis is in a direction of the seam dip. The direction of $x$-axis is chosen in a way that the coordinate triedar is right.

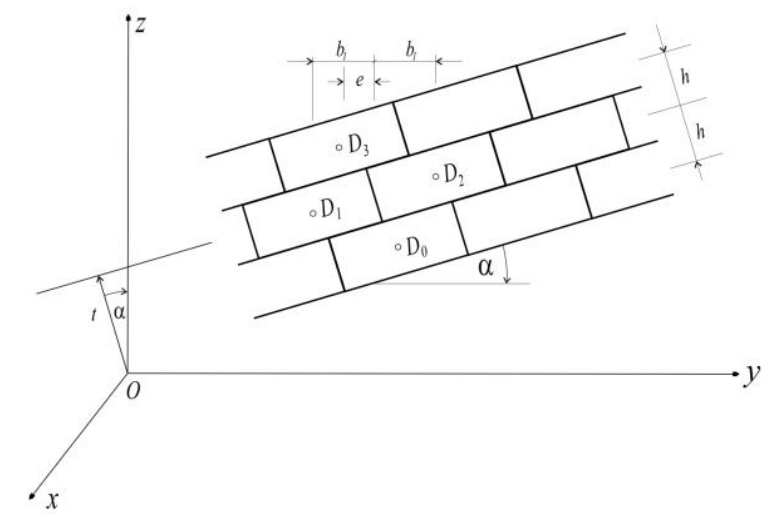

Figure 3 Moving process in the massif with inclined seams [3] 
The equation of the plane of the seam at the distance $t$ from the coordinate origin is:

$$
t=z \cos \alpha-y \sin \alpha
$$

In this case, the place of the lowest removed element $D_{0}$ will be occupied by the element $D_{1}$ or by the element $D_{2}$, but owing to the disturbed symmetry, the probabilities of these events are not equal. Their values can be calculated as the resistances of simply supported beam of span $2 b_{1}$ loaded with a unit eccentric force, so the probability is determined as follows [3]:

$$
\begin{aligned}
& \left(\frac{1}{2}+\frac{e}{2 b_{1}}\right) F\left(x, y+b_{1}, t\right)+\left(\frac{1}{2}-\frac{e}{2 b_{1}}\right) \\
& F\left(x, y-b_{1}, t\right)=F(x, y-e, t+h)
\end{aligned}
$$

The massif is assumed to be anisotropic, therefore the subsidence curves in the profiles by the seam strike are symmetrical, and asymetrical in the profiles by the seam stike [3].

When deriving the equations, it is assumed that the centers of elements remain in the same vertical plane during subsidence. In the general case, the center of element during the subsidence will move both by the strike and dip, so the function of subsidence probability $F$ depends on all coordinates. Due to the independence of the influence of individual coordinates, the equations:

$$
\begin{aligned}
& F(x, \bar{x}, y, \bar{y}, z, \bar{z})= \\
& \quad=F(x, \bar{x}, t, \bar{t}) F(y, \bar{y}, t, \bar{t})
\end{aligned}
$$

represents the total subsidence probability for inclined seams [3].

\subsection{Basic formulas for subsidence calculation of the horizontal seam}

When excavating layered deposits, the used excavation methods that remove the rectangular excavation panels of the layer, whereby the sides of rectangle are parallel to the coordinate axes- $O_{\xi}$ by the seam strike and $O_{\eta}$ by the seam dip.The subsidence in a point $M(x, y, H)$ of the terrain surface $z=H$ of the undermined massif based on the Eq. (7) will be a double integral:

$$
U(x, y)=\iint_{\Omega_{2}} F(x, \bar{x}, y, \bar{y}, z, \bar{z}) d \bar{x} d \bar{y} d \bar{z}
$$

If the sides of the excavation panel $\Omega_{2}$ are $2 a$ by the seam strike and $2 l$ by the seam dip, the coordinate origin is at the intersection point of the rectangle diagonals will be: $-a \leq \xi \leq a ;-b \leq \eta \leq b$ and using the function:

$$
\Phi(x)=\frac{2}{\sqrt{2 \pi}} \int_{0}^{x} e^{-\frac{1}{2} t^{2}} d t
$$

a definite formula for calculating the $s u b$ sidence during horizontal seam excavation is obtained [3].

$$
U(x, y)=U_{0} X(x) Y(y)
$$

where:

$$
\begin{aligned}
& X(x)=\frac{1}{2}\left[\Phi\left(p \frac{a+x}{\sqrt{H}}\right)+\Phi\left(p \frac{a-x}{\sqrt{H}}\right)\right] \\
& Y(y)=\frac{1}{2}\left[\Phi\left(p \frac{l+y}{\sqrt{H}}\right)+\Phi\left(p \frac{l-y}{\sqrt{H}}\right)\right]
\end{aligned}
$$

The function $\Phi$ from expression (9) represents the standard Gaussian distribution curve.

\subsection{Basic formulas for subsidence calculation of the inclined seam}

If during the excavation of inclined layers, the exploitation area $\left(\Omega_{3}\right)$ is of arbitrary shape, the total subsidence $M(x, y, H)$ on the surface of the undermined massif based on (7) will be, 


$$
U(x, y)=\iiint_{\Omega_{3}} F(x, \bar{x}, y, \bar{y}, H, z) d \bar{x} d \bar{y} d \bar{z}
$$

If the seam dip angle $(\alpha)$ and the seam thickness $(d)$ are constant, the area $\Omega_{3}$ is prismatic with the base $\Omega_{2}$ (Figure 4).

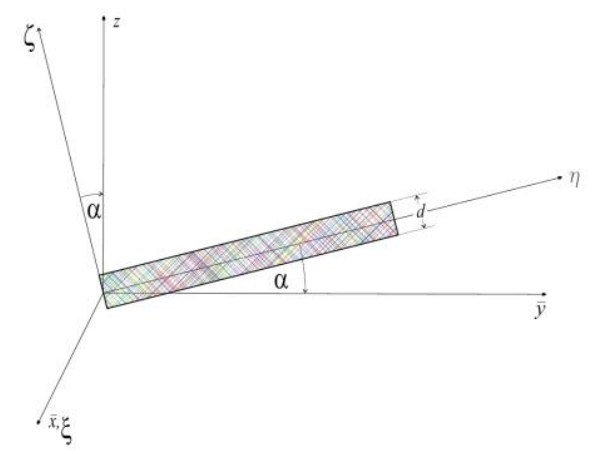

Figure 4 Plane subsidence of inclined seam [3]

By introducing the new variables: $\bar{x} \equiv \xi$ ; $\bar{x}=\xi ; \bar{z}=\eta \sin \alpha-\zeta \cos \alpha$, the subsidence calculation can be simplified, because if $\xi=0, \bar{y}=\eta \cdot \cos \alpha$ and $\bar{z}=\eta \cdot \sin \alpha$, the difference when $z=H$ is:

$$
\begin{aligned}
t-\bar{t} & =(z-\bar{z}) \cdot \cos \alpha-(y-\bar{y}) \cdot \sin \alpha= \\
& =H \cdot \cos \alpha-y \cdot \sin \alpha
\end{aligned}
$$

and no longer depends on the variable $\eta$.
If the sides of the excavation panel $\Omega_{2}$ are $2 a$ by the seam strike and $2 l$ by the seam dip, with the coordinate origin at the intersection point of diagonals of the rectangle it will be: $-a<\xi<a,-l<\eta<l$, the expression for calculating the subsidence during inclined seams excavation is obtained [3]:

$$
U(x, y)=U_{0} X(x, y) Y(y)
$$

where:

$$
\begin{aligned}
& X(x, y)=\frac{1}{2}\left[\Phi\left(p \frac{a+x}{\sqrt{H \cdot \operatorname{ctg} \alpha-y}}\right)+\Phi\left(p \frac{a-x}{\sqrt{H \cdot \operatorname{ctg} \alpha-y}}\right)\right] \\
& Y(y)=\frac{1}{2}\left[\Phi\left(q \frac{b+m+y}{\sqrt{H \cdot \operatorname{ctg} \alpha-y}}\right)+\Phi\left(q \frac{b-m-y}{\sqrt{H \cdot \operatorname{ctg} \alpha-y}}\right)\right]
\end{aligned}
$$

where:

$$
\begin{aligned}
& p=\frac{p_{0}}{\sqrt{\sin \alpha}}, q=(1-\lambda \sin \alpha) Q, \\
& b=\frac{l \cos \alpha}{1-\lambda \sin \alpha}, m=\frac{H \lambda \cos \alpha}{1-\lambda \sin \alpha}
\end{aligned}
$$

The subsidence curve will be the same in any profile by the dip, so it is a plane subsidence:

$$
U(y)=U_{0} Y(y)
$$

where $Y(y)$ is calculated from the formula (12). 
In the case that $\alpha=0$,the formula (12) is reduced to the basic formula (10) for calculating the subsidence of horizontal seam.

\subsection{Geometric interpretation of parameters for the subsidence predictive calculation}

The subsidence calculation formulas based on the stochastic method, formulate two types of parameters:

- Parameters $a, l, H, d, \alpha, b$ that are known and determined;

- Parameters $U_{0}, p, m, q, \delta$ that are not determined in the physical sense. These parameters describe the behavior of an undermined rock massif during consolidation and need to be determined previously to find their relationship with some unit that can be measured.

The following terms, definitions, and symbols are adopted:

Dimensions of the excavation panel $a, l[\mathrm{~m}]$ are determined from the project and depend on the exploitation area.

Seam depth $(H)$ represents the mean seam depth for each excavation panel. It is calculated from:

$$
H=H_{T}-H_{S}
$$

where $H_{T}$ is the elevation of terrain over the excavated seam [m] and $H_{S}$ is the depth of excavation panel [m].
Angle of seam dip $\alpha\left[^{\circ}\right]$ varies. For the predictive calculation of the movement parameters, the mean values of the seam dip for each excavated area are taken.

Maximum subsidence $U_{0}[\mathrm{~mm}] \mathrm{de}-$ fines the maximum possible subsidence (Figure 5) that can occur at the full excavation surface. Only one point $M$ at the ground surface has a maximum possible subsidence. It is calculated from the expression [3]:

$$
U_{0}=d \cdot q_{u} \cdot i \cdot \cos \alpha
$$

where: $q$ is the rate of caving represents the coefficient of subsidence of the hanging wall and is taken as a real value based on the analysis of former excavation. The most commonly adopted value is $q=0.7-0.9$ which corresponds to the value that would be taken based on an analogy from a basin with the similar physico-mechanical properties of a hanging wall. The parameter $\mathrm{q}$ is calculated from the expression:

$q=\frac{f+2}{\sqrt{300 \sin \alpha}} \exp [(0,0027-0,0076 \sqrt{f}) \alpha]$

where: $f$ - is a strength coefficient of the rock massif by Protodjakonov; $i$ - coal deposit recovery is determined with losses $g(\%)$, which corresponds to the designed excavation method, whereby $i=1-g$.

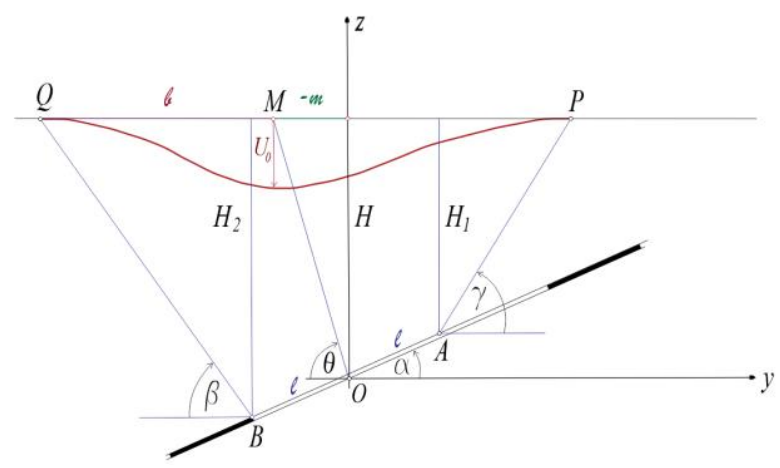

Figure 5 Parameters for mine subsidence prediction [4] 
Parameter $p\left[\mathrm{~m}^{-1}\right]$ depends on the relationship between the side $a$ and the hypothetical element $h$. It is calculated from the expression:

$$
p=\frac{f+2}{\sqrt{300 \cdot \operatorname{tg} \alpha}} .
$$

Parameter $m$-to determine this parameter, it is necessary to know the position of the point with the maximum subsidence $U_{0}$ which is in the point $M$, with the ordinate $Y_{M}=-m$. The parameter $m$ is in direct connection with the angle of full subsidence $\theta$ (Figure 5), which is related with the angle of draw $\delta$, ie. with auxiliary angles $\beta$ and $\gamma$. Parameter $m$ is calculated according to the empirical formula:

$$
m=H \operatorname{ctg} \theta
$$

Parameter $b$ is not independent and is calculated from the known data: the seam $\operatorname{dip} \alpha$, dimension of the excavation field $l$, seam depth $H$ and parameter $m$ (Figure 5). It can be expressed through the angle $\theta$ and calculations according to an empirical formula:

$$
b-l \cos \alpha=\frac{m}{H} \sin \alpha
$$

by entering the value $m=H \operatorname{ctg} \theta$ it will be: $b-l \cos \alpha=l \sin \alpha \operatorname{ctg} \theta$, so the parameter $b$ is calculated from the expression:

$$
b=l \frac{\sin (\alpha+\theta)}{\sin \theta}
$$

Angles of draw $\left(\delta_{1}, \beta_{1}, \gamma_{1}\right)$ are angular characteristics of the moving process that determine the ground surface area affected by the displacements caused by excavation, assuming that the deformations can be neglected beyond that displacement boundary. These are angles between the horizontal and line joining the excavation boundary in the pit with some boundary point at the land surface (Figure 6).

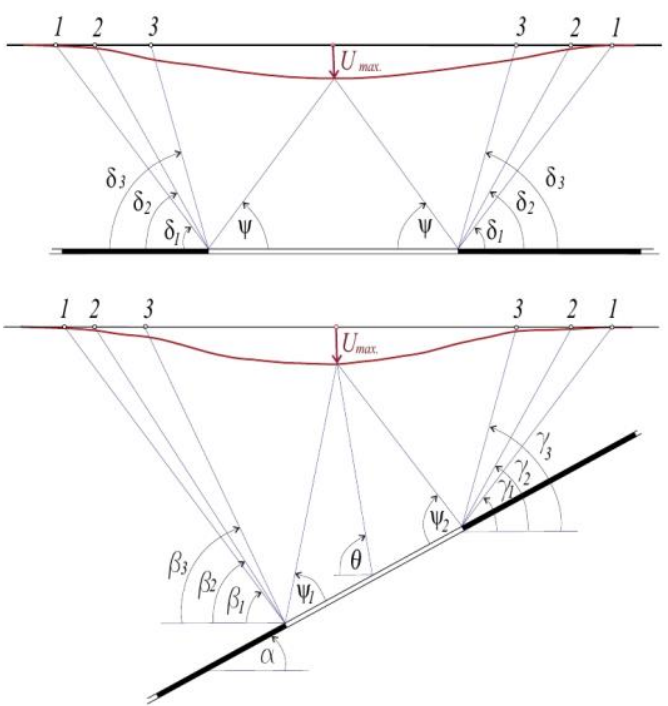

Figure 6 Angular parameters of undermined ground movement process:

$\delta_{1}, \beta_{1}, \gamma_{1}$-angles of the draw; $\delta_{2}, \beta_{2}, \gamma_{2}$-safety angles; $\delta_{3}, \beta_{3}, \gamma_{3}$-fracture angles;

$\psi, \psi_{1}, \psi_{2}$-full movement angles; $\theta$ - angle of full subsidence; $\alpha$ - angle of seam dip [5] 
The boundary point is defined by the value of measured displacement. Both components of the total displacement vector (horizontal and vertical), which have the millimeter values on the peripheral part, can be measured. The boundary point is easier to determine through the vertical component -subsidence. The absolute value of the subsidence $U_{g}=10 \mathrm{~mm}$ is adopted for the boundary condition. For predictive calculations, there is a problem of adopting the draw angles. For that purpose, Table 1 was created, in which the values are given based on the interdependence of angles: $\delta_{1}, \beta_{1}, \gamma_{1}, \theta[4]$.
The values of draw angles in the directions $\beta_{1}$ and $\gamma_{1}$ are different. The subsidence curve is asymmetric with $U_{\max }$ shifted in the direction the seam dip $\alpha$ with the angle of maximum subsidence $\theta$. When the coordinates of the points $P$ and $Q$ (Figure 5):

$$
\begin{aligned}
& y_{P}=l \cdot \cos \alpha+H_{1} \cdot \operatorname{ctg} \gamma \\
& y_{Q}=-l \cdot \cos \alpha-H_{2} \cdot \operatorname{ctg} \beta
\end{aligned}
$$

are entered into the Eq. (13), the subsidences in these points are obtained [3]:

$$
\begin{aligned}
& U_{P}=\frac{U_{0}}{2} \cdot\left[\Phi\left(q \cdot \frac{b+m+l \cdot \cos \alpha+H_{1} \cdot \operatorname{ctg} \gamma}{\sqrt{H \cdot \operatorname{ctg} \alpha-l \cdot \cos \alpha-H_{1} \cdot \operatorname{ctg} \gamma}}\right)+\Phi\left(q \cdot \frac{b-m-l \cdot \cos \alpha+H_{1} \cdot \operatorname{ctg} \gamma}{\sqrt{H \cdot \operatorname{ctg} \alpha-l \cdot \cos \alpha-H_{1} \cdot \operatorname{ctg} \gamma}}\right)\right] \\
& U_{Q}=\frac{U_{0}}{2} \cdot\left[\Phi\left(q \cdot \frac{b+m-l \cdot \cos \alpha-H_{2} \cdot \operatorname{ctg} \beta}{\sqrt{H \cdot \operatorname{ctg} \alpha+l \cdot \cos \alpha+H_{2} \cdot \operatorname{ctg} \beta}}\right)+\Phi\left(q \cdot \frac{b-m+l \cdot \cos \alpha+H_{2} \cdot \operatorname{ctg} \beta}{\sqrt{H \cdot \operatorname{ctg} \alpha+l \cdot \cos \alpha+H_{2} \cdot \operatorname{ctg} \beta}}\right)\right]
\end{aligned}
$$

\begin{tabular}{|c|c|c|c|c|c|c|c|c|c|c|c|c|}
\hline \multicolumn{2}{|c|}{ Angle of draw } & \multicolumn{7}{|c|}{ Theory is valid, stable foot wall } & \multicolumn{4}{|c|}{ Theory is not valid, active foot wall } \\
\hline$f$ & $\mathrm{a}=0^{\circ}$ & $\mathrm{a} \neq 0^{\circ}$ & $\mathrm{a}=10^{\circ}$ & $\mathrm{a}=20^{\circ}$ & $\mathrm{a}=30^{\circ}$ & $\mathrm{a}=40^{\circ}$ & $\mathrm{a}=50^{\circ}$ & $\mathrm{a}=55^{\circ}$ & $\mathrm{a}=60^{\circ}$ & $\mathrm{a}=70^{\circ}$ & $\mathrm{a}=80^{\circ}$ & $\mathrm{a}=90^{\circ}$ \\
\hline \multirow{3}{*}{1.5} & \multirow{3}{*}{$\delta_{1}=55^{\circ}$} & $\beta_{1}$ & 47 & 42 & 37 & 33 & 30 & 29 & 28 & 27 & 26 & 25 \\
\hline & & $\gamma_{1}$ & 57 & 60 & 64 & 67 & 71 & 73 & 73 & 57 & 40 & 25 \\
\hline & & $\theta$ & 85 & 81 & 78 & 76 & 74 & 74 & 73 & 75 & 80 & 90 \\
\hline \multirow{3}{*}{2.5} & \multirow{3}{*}{$\delta_{1}=60^{\circ}$} & $\bar{\beta}$ & 53 & 47 & 41 & 37 & 33 & 32 & 29 & 28 & 27 & 26 \\
\hline & & $\gamma_{1}$ & 63 & 66 & 69 & 71 & 74 & 75 & 76 & 59 & 41 & 26 \\
\hline & & $\theta$ & 85 & 81 & 77 & 75 & 73 & 73 & 71 & 73 & 79 & 90 \\
\hline \multirow{3}{*}{4.0} & \multirow{3}{*}{$\delta_{1}=65^{\circ}$} & $\overline{\beta_{1}}$ & 59 & 52 & 46 & 40 & 46 & 34 & 32 & 29 & 28 & 27 \\
\hline & & $\gamma_{1}$ & 70 & 72 & 74 & 76 & 77 & 78 & 79 & 61 & 42 & 27 \\
\hline & & $\theta$ & 84 & 80 & 76 & 73 & 72 & 71 & 72 & 70 & 77 & 90 \\
\hline \multirow{3}{*}{5.0} & \multirow{3}{*}{$\delta_{1}=70^{\circ}$} & $\overline{\beta_{1}}$ & 62 & 54 & 47 & 42 & 37 & 35 & 33 & 32 & 31 & 29 \\
\hline & & $\gamma_{1}$ & 73 & 75 & 78 & 79 & 81 & 83 & 82 & 64 & 43 & 28 \\
\hline & & $\theta$ & 83 & 77 & 72 & 69 & 67 & 66 & 67 & 70 & 77 & 90 \\
\hline \multirow{3}{*}{8.0} & \multirow{3}{*}{$\delta_{1}=75^{\circ}$} & $\overline{\beta_{1}}$ & 63 & 53 & 46 & 40 & 36 & 34 & 33 & 32 & 31 & 29 \\
\hline & & $\gamma_{1}$ & 78 & 80 & 83 & 84 & 85 & 86 & 85 & 67 & 44 & 29 \\
\hline & & $\theta$ & 82 & 76 & 71 & 67 & 66 & 65 & 66 & 69 & 76 & 90 \\
\hline
\end{tabular}

These are the general conditions for the massif inclined seams.

Table 1 Prediction values of draw angles $\left(\delta_{1}, \beta_{1}, \gamma_{1}\right)$ and maximum subsidence angle $(\theta)$ [4] 


\section{DEVELOPMENT OF THE GIS BASED STOCHASTIC METHOD FOR THE MINE SUBSIDENCE PREDICTION}

Since the subsidence prognosis using equations of the stochastic PataricStojanovic method would be very complicated and time-consuming in case of manual data processing, the original computer program package with the title MITSOUKO has been created based on this method and formulas. MITSOUKO is designed in the Python programming language and provides the mine subsidence and deformations calculation, based on the simulating excavation proces according to the adopted dynamics, at any point of the land surface and representing the results owing to their integration and further processing in the GIS. It consists of three modules: PARAMETERS, SUBSIDENCE and DEFORMATIONS which represent the individual independent functions. Each module starts with form according to a textual description explaining its name and function, the tags of input data that are loaded (Read parameters) or computed (Calculate parameters) into a particular module, and the data values returned by a module through the control loop. The modules have control functions, descibing what the program does according to the algorithm shown in Figure 7.

Firstly, by entering the MITSOUKO program, in the PARAMETERS module, the function given by Eq. 9 is initialized [3]. Then, through the menu, the data obtained based on geometric characteristics for each excavation panel are entered: $I D$, dimensions $(a, l)$, seam depth $(H)$, seam thickness $(d)$ and seam dip angle $(\alpha)$. In the next step, in a specified subroutine of the PARAMETERS module, the following is calculated for each panel: maximum subsidence $\left(U_{0}\right)$, parameters $(m, b, p)$, rate of caving ( $q$ ), angles of draw $(\delta, \beta, \gamma)$, and angle of full subsidence $(\theta)$.

The local coordinate system is situated symmetrically with respect to the first excavation panel, with $x$-axis in the direction of the seam strike, $y$ - axis in the direction of the seam dip and the coordinate origin in the diagonal intersection of this panel. The positions of all panels $\left(x_{i}, y_{i}, F_{i}\right)$ are determined with respect to the defined local coordinate system. During the calculation for each panel, it is necessary to rotate its coordinate axes for the value of angle $F_{i}$ (expressed in degrees) to the direction of axes of the local coordinate system.

Subsidences are calculated in the SUBSIDENCE module. A certain subroutine allows entering the coordinates of points in a grid of a given density, through the assigned distances between points ( $\Delta x, \Delta y)$, in the $x$ and $y$ axes directions of the local coordinate system. In this way, it is possible to define the calculation limits for all panels up to a limit subsidence value of $10 \mathrm{~mm}$. Further, the subsidence values after mining each panel are calculated cumulatively, according to the projected mining dynamics.

The individual results for each panel can be exported (write) in tabular form in an Excel file, which is also the preparation for graphical presentation and spatial analysis in GIS.

The DEFORMATIONS module at the given points of the defined excavation panels calculates and presents in tabular form in an Excel file the $x, y$ components of the tilt, and the directions of the main tilt due to 
the mining out all panels. First, the components of the main tilt, formed by mining one panel, are calculated in the direction of the absolute coordinate system, and then for all panels, as in the subsidence calculation. The components of the total tilt in the direction of the absolute coordinate system axes at a given point are obtained by summing the slope components of the individual panels. Based on these components, the main tilt and its direction are calculated. The program provides calculating curvatures for the given profile points in tabular form in an Excel file and their graphical presentation in the direction of any section after mining out each panel.

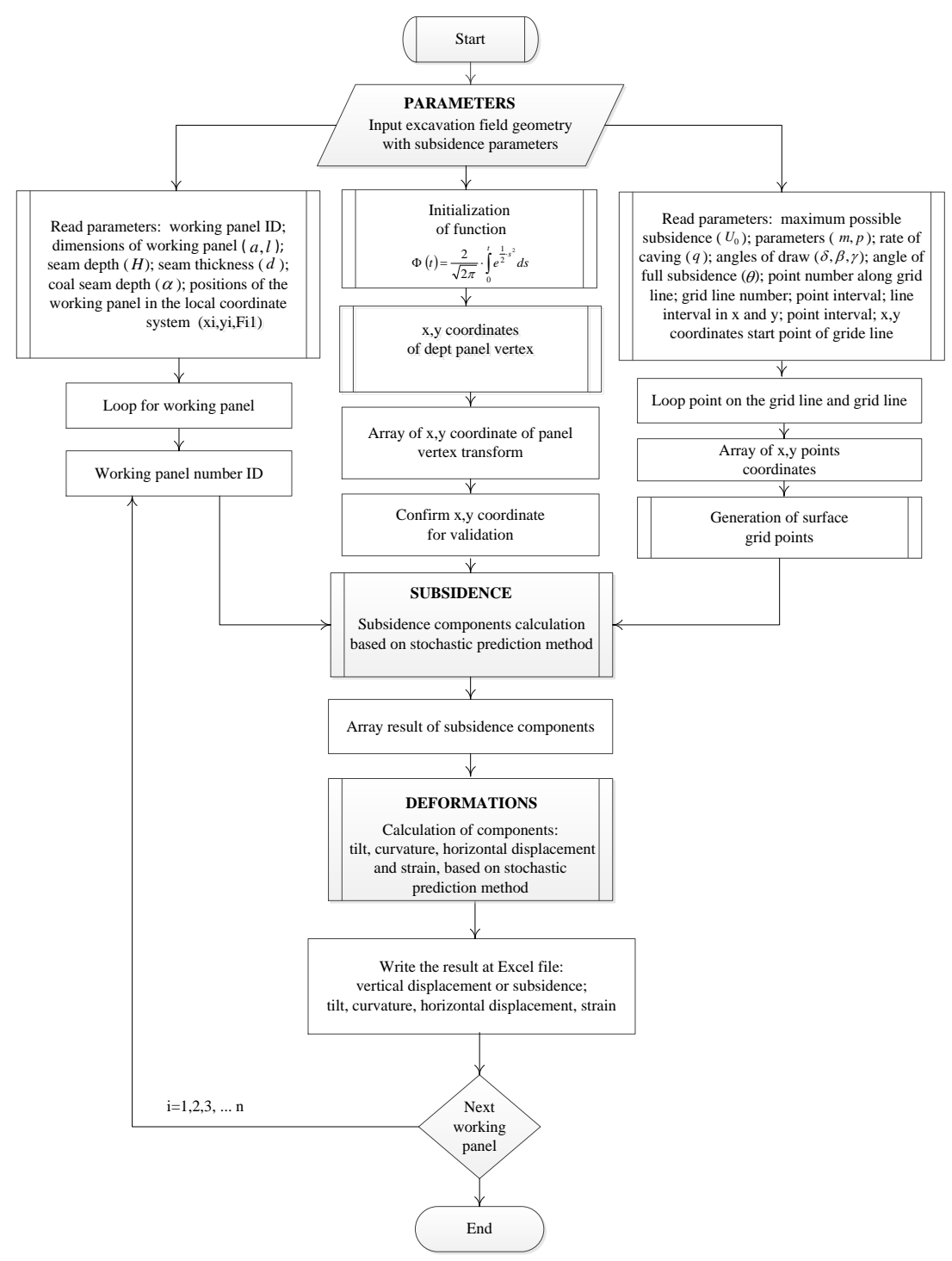

Figure 7 Stochastic prediction algorithm for the subsidence and deformations calculation using the MITSOUKO software 
The program further calculates the curvature radius, as the reciprocal value of curvature in the interval, after mining each panel. Also, the values of horizontal displacements and strains caused by mining all panels are calculated and tabulated.

GIS is used for creatinh complex geodatabase, converting numerical data, imported from the SUBSIDENCE and DEFORMATIONS modules, in feature classes and graphical data as well as for performing the spatial analyses of subsidence and deformations [7-11]. Since the pit consists of multiple panels with complex geometry, spatial analyses requires a long time without GIS because for each panel subsidence or deformations must be presented cumulatively. Implementing the stochastic method for spatial analyses of subsidence and deformations in the GIS is performed in three steps:

The first step, Data module, involves creating a geodatabase of the coal mine in ArcCatalog, within the ArcGIS application (ESRI Software Inc. http://www.esri.com/ software/arcgis/) with feature classes, tables, and rasters. The feature class is a set of homogeneous spatial attributes in the form of digitized vector data, in the same National Coordinate System (MGI Balkans7). In order to integrate the feature classes thematically and spatially into the mine model, within the given excavation panels, feature datasets have been created, in which all types of feature classes are entered. Feature datasets with the feature classes related to the spatial geometry: terrain topographies, buildings, mining facilities, old mining works and new exploitation area in pit, excavation panels with mining dynamics, active and old mining premises, and geological interpretation of the coal seam are created in geodatabase of mine. Outside the feature datasets, tables with subsidence, tilt, curvature, horizontal displacement and strain, calculations from the SUBSIDENCE and DEORMATIONS module in the MITSOUKO program (Figure 8), rasters for the subject area in the form of orthophoto, geographic maps, situational plans of mine and photographs are imported in the geodatabase. Feature Datasets have been created, in which all types of feature classes are entered. Also, using ArcMap, an integrated part of the ArcGIS software package, to create layers for displaying feature classes from the ArcCatalog (ESRI) is included. All tables of the subsidence calculations have been transferred from the geodatabase coal mine to the ArcMap.

The second step, Subsidence module, involves using the Display XY Data command, the selected table of the excavation panel, which contains the $x, y$ coordinates of the points and the calculated subsidence values, is added as a new layer in the Table of Contents. Thereby, a new feature class, panel ID_events, was formed, which for panel contains many points with values of $x$ and $y$ coordinates and associated subsidences, georeferenced to the adopted coordinate system MGI Balkans7. Following the same procedure, new feature classes were created for all panels. The created feature classes contain $x$ and $y$ coordinates of all points in the grid 10x10 m with associated subsidence values. The Spline interpolation method from the Spatial Analyst Tools palette is then used to create new layers with contour subsidence lines for each panel, by the cumulative subsidence transformation from the previous to the new state. This provides successive following of the subsidence process on the map at all mining stages. Digitization and formation of feature classes for all panels in the global MGI Balkans7 coordinate system are performed in a map. 


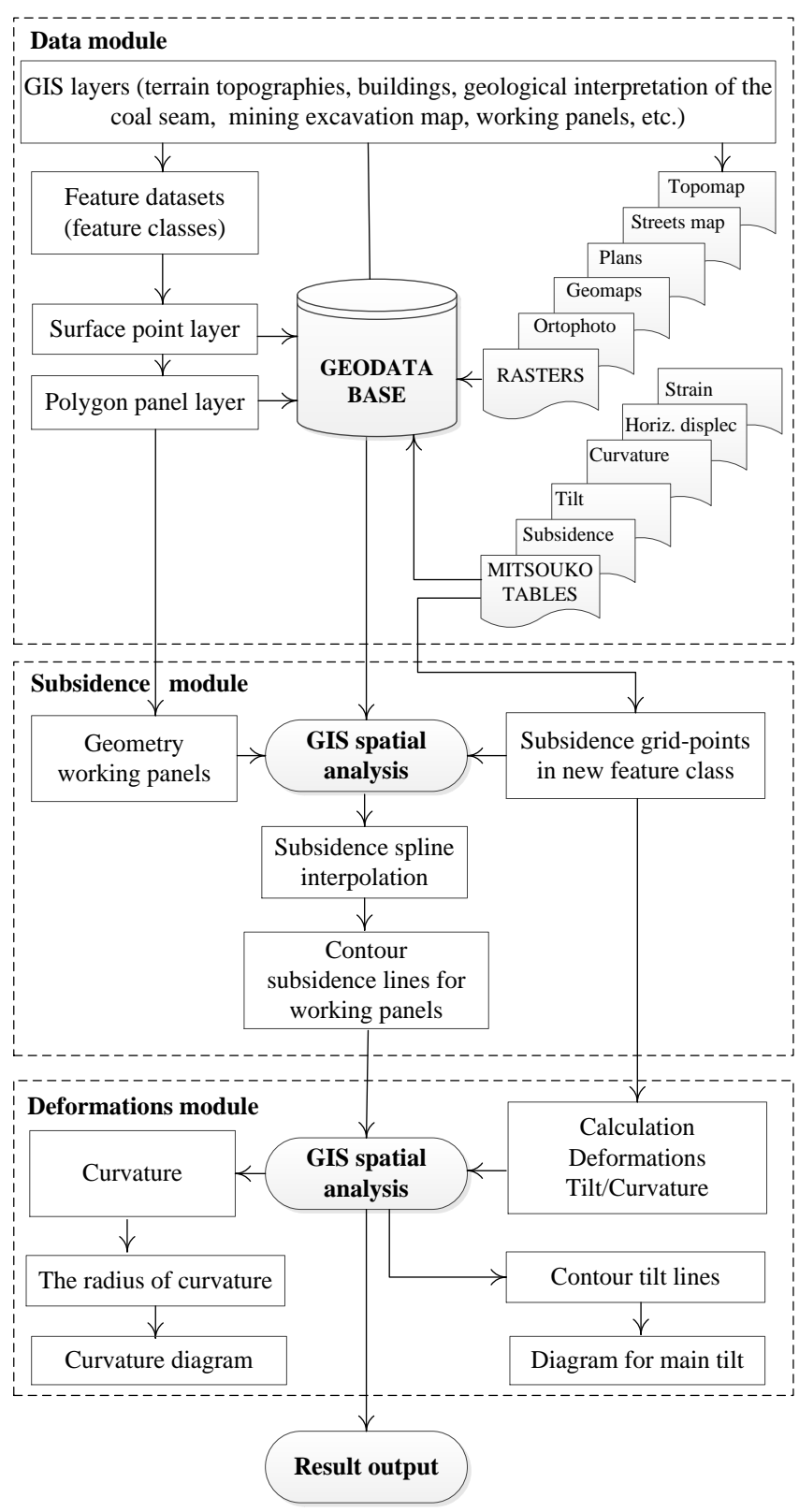

Figure 8 Algorithm connecting stohastic prediction analysis with the GIS spatial analysis function 


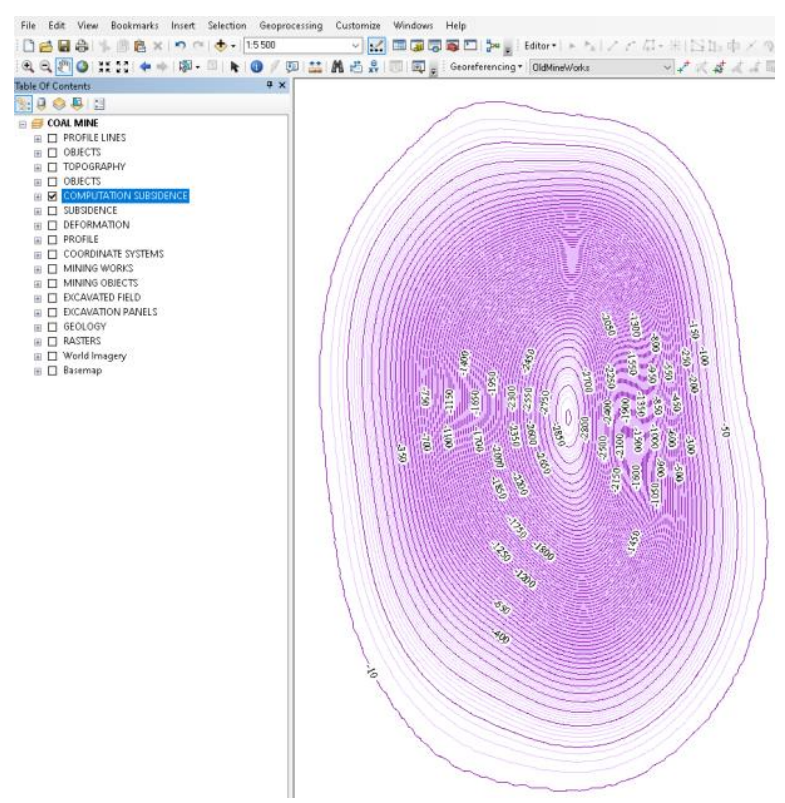

Figure 9 Interpolation of subsidence contour lines after mining excavation panels

In the third step, Deformations module, based on the subsidence values for individual panels obtained in the GIS spatial analysis, tilt is calculated cumulatively as subsidence increment after mining each panel.
The Spline or Kriging interpolation method from the Spatial Analyst Tools palette is then used to create the new layers with the contour tilt lines (Figure 10). It is possible to draw the tilt diagrams and the main tilt according to a given profile.

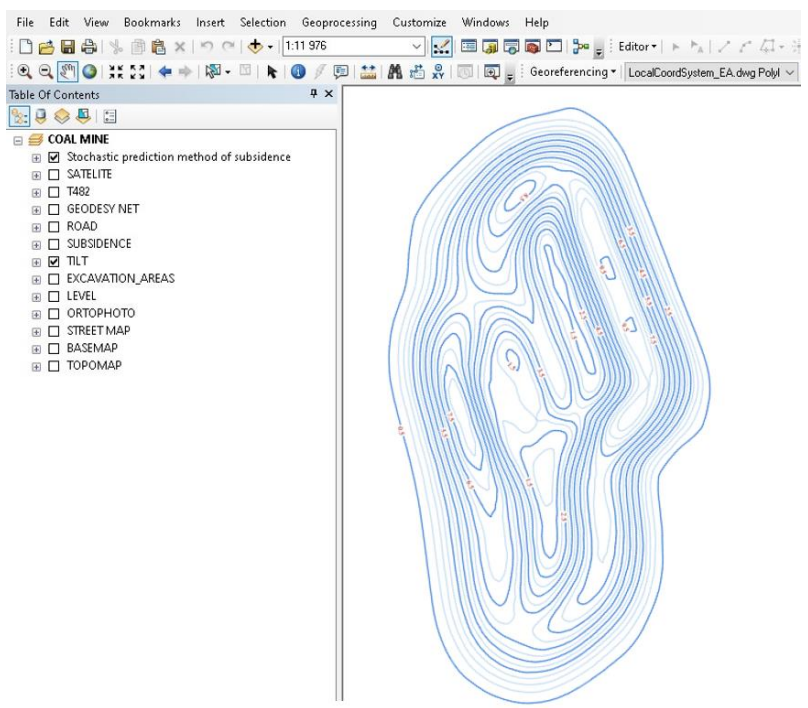

Figure 10 Interpolation of tilt lines after mining out all excavation panels 
Based on the subsidence increment, after mining each panel, for the profile points, the curvature is calculated and presented, i.e. the curvature radius as its reciprocal value.

\section{CONCLUSION}

The calculation of subsidence by the stochastic Pataric-Stojanovic method and originally developed MITSOUKO program package integrated with the GIS are powerful tools for predicting subsidence and monitoring the impact of underground mining works on the land surface.

Benefits of this approach are:

- reliability of the method for calculation of subsidence and deformations for inclined seams;

- equations for inclined seams, in cases when $\alpha \rightarrow 0^{\circ}$, give solutions for horizontal seams;

- definition of draw angles in terms of the possibility of their application when seam thickness and excavation depth increase, and

- mutual connection between the angular parameters of the displacement process.

\section{REFERENCES}

[1] Kratzsch H., Mining Subsidence Engineering, Springer-Verlag, Berlin, Germany, 1983.

[2] Litwiniszyn J., The Theories and Model Research of Movements of Ground Mass. Proceedings of the European Congress on Ground Movement, Leeds, 1957, 202-209

[3] Patarić M, Stojanović A., Movement of Undermined Terrain and Protection of Structures on Mining Activities, University in Belgrade, MGF, Belgrade, 1994 (in Serbian)
[4] Đorđević D, Vušović N., Forecast Calculation of Movement and Deformations of the Undermined Terrain, University in Belgrade, MGF, Belgrade, 2014 (in Serbian)

[5] Đorđević D, Vušović N, Ganić A, Svrkota I., Angular Parameters of Undermined Ground Movement Process in Unknown Areas, University of Belgrade, Faculty of Mining and Geology, Underground Mining Engineering, 19 (2011) 125-136.

[6] Burrough PA, McDonnel RA., Principles of Geographical Information Systems. $2^{\text {th }}$ ed. Oxford, London, 2006.

[7] Jiayuan C., Fengshan M., Jie G., Rong L., Guowei L., Assessment of MiningRelated Seabed Subsidence Using GIS Spatial Regression Methods: A Case Study of the Sanshandao Gold Mine (Laizhou, Shandong Province, China), Environmental Earth Sciences 78(1)(2019) 26-37

[8] Malinowska A, Hejmanowski R., Evaluation of Reliability of Subsidence Prediction Based on Spatial Statistical Analysis, International Journal of Rock Mechanics and Mining Sciences 46 (2009) 432-438.

[9] Blachowski J., Application of GIS Spatial Regression Methods in Assessment of Land Subsidence in Complicated Mining Conditions: Case Study of the Walbrzych Coal Mine (SW Poland), Natural Hazards, 84 (2016) 997-1014

[10] Petrović V, Borisov M, Zlatanović D, Djurdjevac Ignjatović L., Vertical Accuracy of Data on the Topographic Maps and their Application in Mining, Mining and Metallurgy Institute Bor No 3-4 (2017) 39-50 
[11] Petrović V, Zlatanović D, Borisov M, Djurdjevac Ignjatović L., Concepts of 3D Terrain Modeling and Geomorphometric Analysis in Mininig, Mining and Metallurgy Institute Bor No. 3 (2016) 1-12
[12] Vušović N, Vuković M, Svrkota I Stojković P., Possibility for Application of GIS Technologies in RTB Bor Group, Mining and Metallurgy Institute Bor No. 3 (2016) 21-30 